\title{
Hard Tissue Reconstruction of the Maxillofacial Region
}

\author{
Srinivasa R. Chandra and Vijay Pillai
}

\section{Abbreviations}

ACS acellular collagen sponge

BFGF basic fibroblast growth factor

BMP bone morphogenic protein

IGF-1,2 insulin-derived growth factor 1,2

PDGF platelet derived growth factor

rh BMP-2 recombinant human bone morphogenic protein-2

TGF- $\beta$ transforming growth factor beta

\subsection{Introduction}

The maxillofacial skeleton can be visualised as a threedimensional structure, and its integrity is essential for neurovisceral function like vision, airway conduction, nutritional luminal support, etc. The location and the size of the defect determine the hard tissue reconstruction and the donor site selection.

\subsection{General Principles [1]}

- The bone graft recipient site should have viable vascular and infection free environment.

- Adequate evaluation of the donor site morbidity has to be undertaken.

Electronic Supplementary Material The online version of this chapter (https://doi.org/10.1007/978-981-15-1346-6_88) contains supplementary material, which is available to authorized users.

S. R. Chandra $(\bowtie)$

Department of Head and Neck Oncology and Reconstructive

Microvascular and Oral and Maxillofacial Surgery, UNMC,

Omaha, NE, USA

Nebraska Medicine Fred \& Pamela Buffett Cancer Center, Omaha, NE, USA

V. Pillai

Department of Head and Neck Surgical Oncology, Mazumdar

Shaw Cancer Center, Narayana Health, Bangalore, India
- Evaluation for vascular versus nonvascularized bone graft has to be done with adequate immobilization and if required vascular anastomosis.

- Comorbidities of the patient and surgical appropriateness have to be prioritized. Smoking, cicatrization, radiation, pain issues and functional status are considerations as well.

\subsection{Principles of Reconstruction}

- Age is a factor but not a contraindication for reconstruction.

- There is no 'ideal reconstruction' but there is 'optimal reconstruction'.

- Patient comorbidities and future adjuvant radiation should be strongly weighed.

- 'Like to like tissue' reconstruction especially hard tissue substitution is optimal.

\subsection{Terminology}

Autograft: Transplant between areas within the same individual.

Allograft: Transplant between individuals (same species).

Xenograft: Transplant between individuals of different species.

Isograft: Transplant between monozygotic twins.

Osteoconduction is the phenomenon of bone regeneration which needs a scaffold for ingrowth of capillaries, osteoprogenitor cells and perivascular tissue. The ingrowth of capillaries from the recipient bed causes differentiation of the osteoprogenitor cells and results in the formation of new bone. There are materials which promote osteoconduction as 
grafts or scaffolds. These can be autologous or alloplastic, homologous, xenogeneic bone particles, bone glass and hydroxyapatite ceramic material.

Osteogenesis is the spontaneous growth of new bone. It can either be spontaneous from progenitor cells in the area of the bone defect, or it could be transplanted osteogenesis arising from grafted progenitor cells.

Osteoinduction is auto induction by differentiation of mesenchymal cells from the native bed into osteoblastic tissue. This requires inductive stimulation, either from the grafted bone material (there is natural bone morphogenetic protein release with the resumption by osteoclastic activity on the middle matrix) or by the addition of recombinant bone morphogenic protein-2. This results in recruitment from the surrounding bed of mesenchymal cells, which then differentiate. This is mediated by graft-derived factors, viz. recombinant human bone morphogenic protein-2 (rhBMP-2), in situ BMP, TGF- $\beta$, IGF-1 and IGF-2, BFGF, PDGF.

\section{Clinical Pearls}

Key concept in bone grafting which is a part of hard tissue reconstruction is the extent of ostial induction, ostial conduction and osteogenesis caused by the graft. The main tenet is that only autologous bone graft grafts can promote osteogenesis due to its volume of biocompetent viable cells. Allo- and xenografts do not have osteogenic potential.

\subsection{Bone Grafts $[2,3]$}

\subsubsection{Cortical vs Cancellous Grafts}

Cancellous grafts revascularize more rapidly when compared to cortical grafts. For example, cranial bone revascularizes more rapidly than other cortical bone.

Mechanism of healing:

- Cancellous: Apposition and subsequent resorption.

- Cancellous grafts repair more completely.

- Cortical bone incompletely resorbed/remodelled

- Cortical: Resorption then apposition. $50 \%$ reduction in strength at 6 weeks- 6 months.

\subsubsection{Considerations in Usage of rhBMP-2 [4]}

In March 2007, the US Food and Drug Administration (FDA) approved the use of rhBMP-2 in maxillofacial surgical procedures for intraoral ridge augmentation.

Biologic concept - the mechanism is through osteoinduction by chemotactic osteoprogenitor cellular proliferation and differentiation causing osteoid maturation.

Technique-BMP is carried in the acellular collagen sponge (ACS) to the defect where the formed osteoid matures into an ossicle about 6 months after the cycle of resorption and remod- elling. There are multiple case reports of allogenic freeze-dried, crushed cancellous bone with BMP and platelet-rich plasma used in large maxillofacial reconstructions. rhBMP-2 is a lyophilized powder mixed with prepackaged sterile water. Concentration of $1.5 \mathrm{mg} / \mathrm{mL}$ of rhBMP-2/ACS is advised. For molecular binding the mixture is kept soaked for 15 minutes. To prevent desiccation and protein loss, rhBMP/ACS mixture must be used within 2 hours. Informed consent, warning of swelling and tension-free closure are important considerations.

Indications: Approved by FDA—spinal fusions (2002), treating open tibial fractures (2004) and oral and maxillofacial sinus and ridge augmentations (2007).

Contraindications and precautions:

- Local health advisory or FDA issued black box warning.

- Pregnancy.

- History of malignancy.

- Active infection.

- Hypersensitivity to BMP or collagen matrix.

- Unknown evidence of safety in children and females of childbearing age.

- Proximity to airway due to severe oedema postoperatively.

\subsubsection{Bone Graft Carriers and Fixation Techniques}

- Mesh.

- Custom fabricated cribs, implants, plates, etc.

- Titanium plates and screws.

- Lag screws.

- Autologous or allogenic bone cribs (Fig. 88.1).

\section{Autogenous Bone Donor Sources}

The choice of donor site depends on:
(a) Quantity.
(b) Quality.
(c) Anatomical contour (height, weight, volume).
(d) Donor site morbidity.
(e) Patient and anatomical access to harvest.
(f) Type of carrier and fixation needed.
(g) Surgical competence.

Common Locations of Autogenous Bone Donor Sites

- Iliac crest (anterior and posterior).

- Costochondral rib graft.

- Cranial bone.

- Tibia.

- Scapula.

- Maxilla.

- Mandible. 

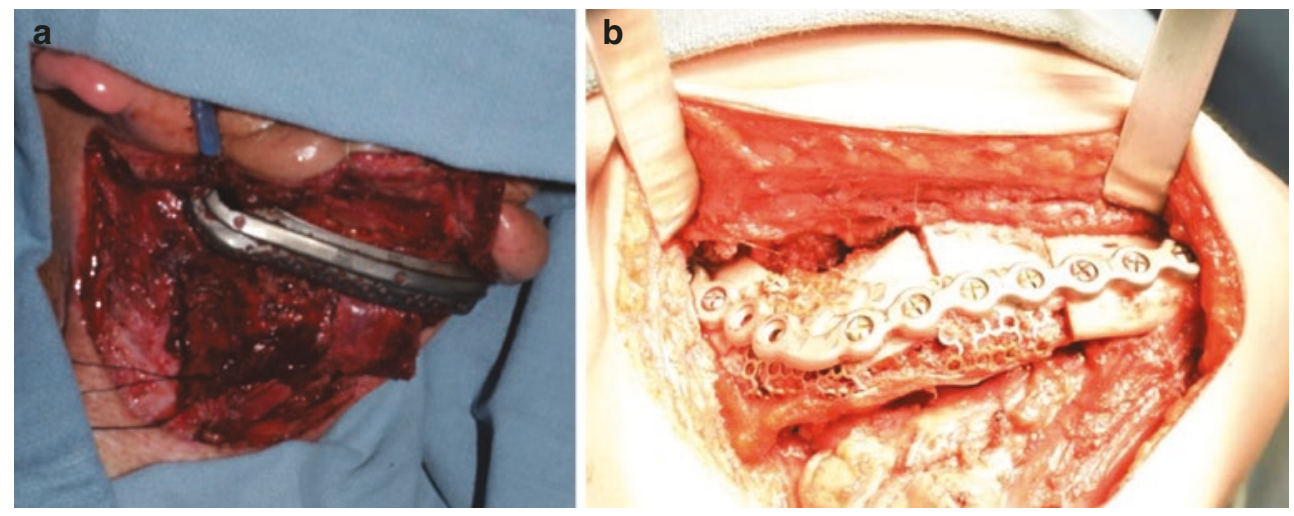

CAssociation of Oral and Maxillofacial Surgeons of India

Fig. 88.1 Bone graft carriers and fixation techniques(a-b) custom made implants compared to stock prosthesis. (a) Custom-built bone graft carrier and plate as patient specific implant-note that this can be planned for optimal areas of fixation (anchoring the crib to the plate, the

\subsubsection{Costochondral Graft}

The costochondral rib graft came into vogue with the concept of transplanting a growth centre to the recipient site in reconstruction. This was initially used for temporomandibular joint arthroplasty and extended to the ramus condyle subunit, as it was a substitute for the growth centre at the condyle and was needed to maintain the growth potential of the joint

\subsubsection{Technique}

A submammary incision is placed around the 5th-seventh rib, taking care not to extend beyond to avoid a flail segment. The incision is made in the mid-clavicular line, placed more medially if a costochondral graft is needed. It is deepened down to bone, and a circumferential subperiosteal dissection is done with a stripper. If the cartilage is harvested, a sleeve of periosteum is retained. Subperiosteal dissection is done avoiding pleural damage. Once the graft is removed, inspect the cavity with saline and check for any air leaks. A layered closure is performed with a drain.

\subsubsection{Indications}

Laryngotracheal reconstruction, nasal reconstruction, auricular reconstruction, facial augmentation in syndromic disorders.

\section{Clinical Pearls}

- Good cartilage yield.

- Superior strength.

\section{Pitfalls}

- Inferior pliability.

- High tendency for warpage.

- Subcostal scar, pneumothorax. span and width of the screws, etc.). (b) The figure shows a reconstruction bar adapted with stock titanium mesh for carrying a non-vascularised iliac bone graf

\subsubsection{Autologous Rib Grafts}

Rib grafts are a good source of corticocancellous membranous bone that can be used as onlay bone grafts for the craniofacial skeleton. Being soft and flexible, it is unable to withstand zones of high stress placement.

The harvest is similar to the costochondral graft; however, leaving the periosteum in situ would allow for regeneration of the rib but would reduce the take of the rib graft.

Pitfalls

- Subcostal scar.

- Pneumothorax.

- Atelectasis.

- Higher incidence of resorption if used as onlay grafts due to the higher cancellous component.

\subsubsection{Iliac Crest}

The ilium is a rich source for cortical, cancellous and a combination of corticocancellous bone grafts.

It is primarily utilized for arthrodesis, bony non-unions and alveolar clefts.

The anatomy of the ilium with the dense cortical bone permits grafts for high stress areas like the long bones and hands and also allows for rigid fixation. The outer table has numerous muscular attachments, thus is not preferred. The inner table is commonly harvested.

The iliac crest allows the harvest of large amounts of cancellous bone. 
The cartilaginous apophysis in children must not be disturbed as it contributes to the normal growth and development.

\subsubsection{Technique}

The anterior approach is the most commonly utilized with the incision being placed after careful palpation to ensure the scar lies on the lateral aspect of the crest.

The incision is deepened through the skin, fascia, muscle and periosteum up to the bone. Ideally the inner or medial table is preferred for harvest as it does not cause disruption of the muscle attachments on the lateral side. The bone cuts should be placed preserving the crest so that it is hinged laterally and can be repositioned once the desired bone has been harvested. Maintaining the periosteal attachments of the crest would preserve its blood supply and further fixation would not be needed.

Good haemostasis, meticulous-layered closure with a drain in the subcutaneous tissue can help prevent a postoperative haematoma. It is also common practice to place an epidural catheter into the bed to permit infusion of a long acting local anaesthetic agent for the first 24 hours.

Most of the disadvantages of iliac bone harvest can be circumvent if the amount of bone graft needed is not too excessive by using the trephination technique.

\section{Pitfalls}

- Postoperative morbidity depending on the extent of bone harvested.

- Injury to the lateral femoral cutaneous nerve with prolonged post- surgical pain.

- Haematoma.

- Gait disturbances.

\subsubsection{Tibia}

\subsubsection{Tibial Autogenous Cancellous Bone Harvest}

This was first reported by Catone [5] in 1992 and is used in maxillofacial bone grafting techniques with a harvest volume of about $25-40 \mathrm{cc}$ from a unilateral site. Harvest can be performed under local or general anaesthesia. The anatomical access to the tibial proximal head can be medial or lateral. Paediatric harvest has been reported, but the growth plate of the tibial plateau should be mature for adequate safety. The contraindications for tibial bone harvest cited are metal prostheses, athletes and history of knee trauma.

\subsubsection{Technique}

As mentioned the approach can be medial or lateral to the midline patellar tendon. In the lateral approach-Gerdy's tubercle is the bony prominence which is palpated between the midline patellar tendon and the lateral proximal head of the fibula. This bony prominence has the attachment of the iliotibial fascial tract. In the medial approach, there is no significant bony prominence, and the only overlying structures to the tibial proximal head are just skin and subcutaneous tissue. Accordingly, an incision on either side goes through the skin and subcutaneous tissue and a self-retaining retractor can be used for retraction.

Once the tibial head is identified, using a narrow drill with a postage stamp technique, a window is created either supra or subperiosteally. This cortex can be left attached to the periosteum and repositioned at the end of the harvest if cortical bone is not needed. Cancellous bone can be curetted with adequate care not to perforate the superior tibial plateau entering the knee joint. Adequate haemostasis with local cautery should be used to prevent any postoperative haematomas. Deep and superficial layer closures are performed. No immobilization is recommended, but active weight bearing is avoided for a couple of days. If there is any concern for superior tibial plateau perforation or knee joint violation, an orthopaedic review is mandated.

\subsubsection{Cranial Bone [2]}

It is well-known through craniofacial surgery that paediatric skull defects less than $1 \mathrm{~cm}$ with an intact pericranium can cause adequate bone healing with osseous regeneration. Calvarial bone grafts can be performed as mono- or bicortical harvest based on the surgeon's competence. Cortical bone shavings are commonly utilized. Just off the midline of the skull sagittal plane, parietal cortical calvarial bone is accessed through skin and the scalp layer incision. Adequate retraction is performed with a self-retaining retractor or assistance. The amount of bone needed is identified in the subperiosteal plane and marked with round bur of a narrow diameter. Adequate outer beveling is performed (as depicted in the figure) using a straight fissure bur for a flat osteotomy technique as parallel to the outer surface. Multiple fissuring osteotomies in a parallel fashion can be performed too. The intracortical medulla is carefully traversed through osteotomes and if any small dural perforations are made, they can be covered with artificial allografts or even haemostatic material. A neurosurgical review is needed in case of dural tear. Haemostasis with bone wax or electrocautery and good pericranial adjacent tissue rotational coverage and closure of the scalp layers leads to reduced postoperative haematomas. The bone harvested can be contoured with a crimping forceps as shown in the figure (Fig. 88.2a and b). 

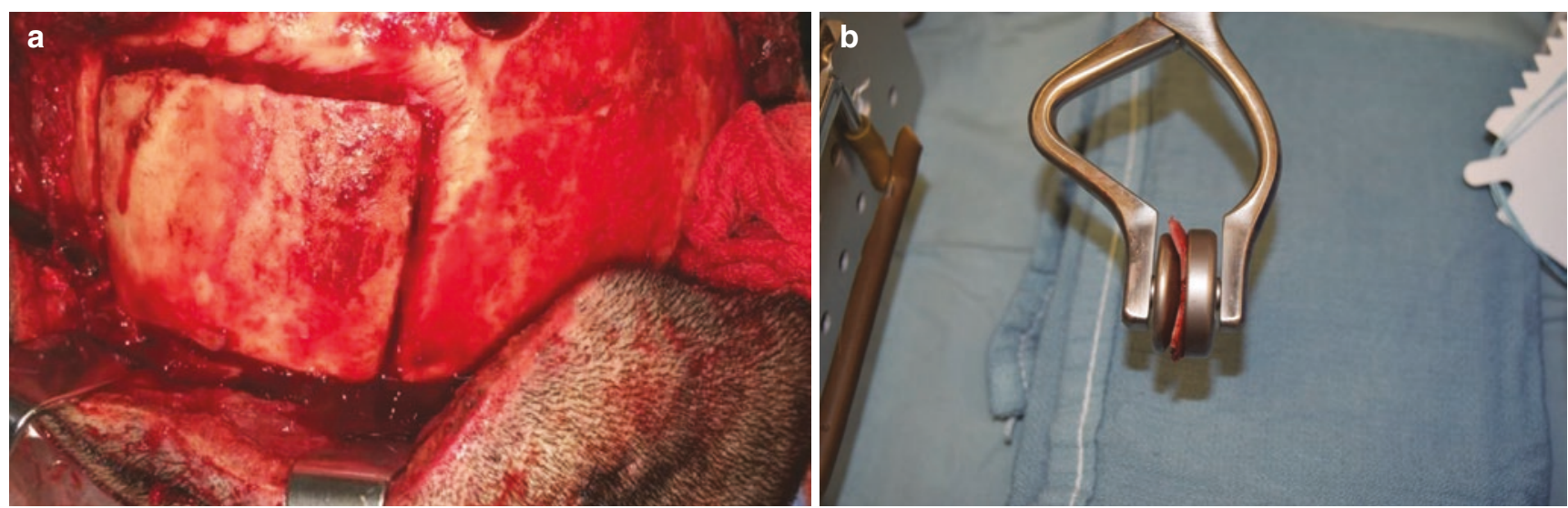

CAssociation of Oral and Maxillofacial Surgeons of India

Fig. $\mathbf{8 8 . 2}$ (a) Cranial bone graft harvest with circumfrential trough and beveling. It is ideal to harvest this in the parietal area. Narrow strips are ideal for harvest. (b) Cranial non vascular bone graft being contoured with pinch forceps

\subsection{Microvascular Free Tissue Transfer}

Most hard tissue grafting using this technique is a 'composite' bone and soft tissue transfer by arteriovenous anastomosis. This transferred bone is usually held in place by plates and/or screws. There is osteogenesis at the interface of the native and grafted bone interface. Current techniques of vascular free tissue using microvascular anastomosis along with minimal donor site morbidity have a success rate close to 98 percent. There is a potential for failure due to anastomotic complication. The vascularized free transferred tissue is resistant to infection and radiation to a degree of volume. There are concepts of creating a vascular envelope and subsequent allogenic or xenogeneic grafting for better osteoconduction by vascular ingrowth.

\subsubsection{Osteocutaneous Radial Forearm Free Flap (OCRFFF)}

The popular workhorse fasciocutaneous radial forearm free flap is very well-known for most reconstructive surgeons. Whereas an osteocutaneous variant of this flap is not the 'go to' flap in composite reconstructions. There is significant donor site morbidity with about $70 \%$ postharvest strength and an increased fracture risk of the distal radius. Distal radius can be reinforced with a prophylactic plate by internal fixation. Figure 88.3 shows osteocutaneous radial forearm free flap for maxillary reconstruction including the orbital rim. Figure $88.4 \mathrm{a}$ and b shows radial forearm flap for reconstruction of maxillary and mandibular defects.

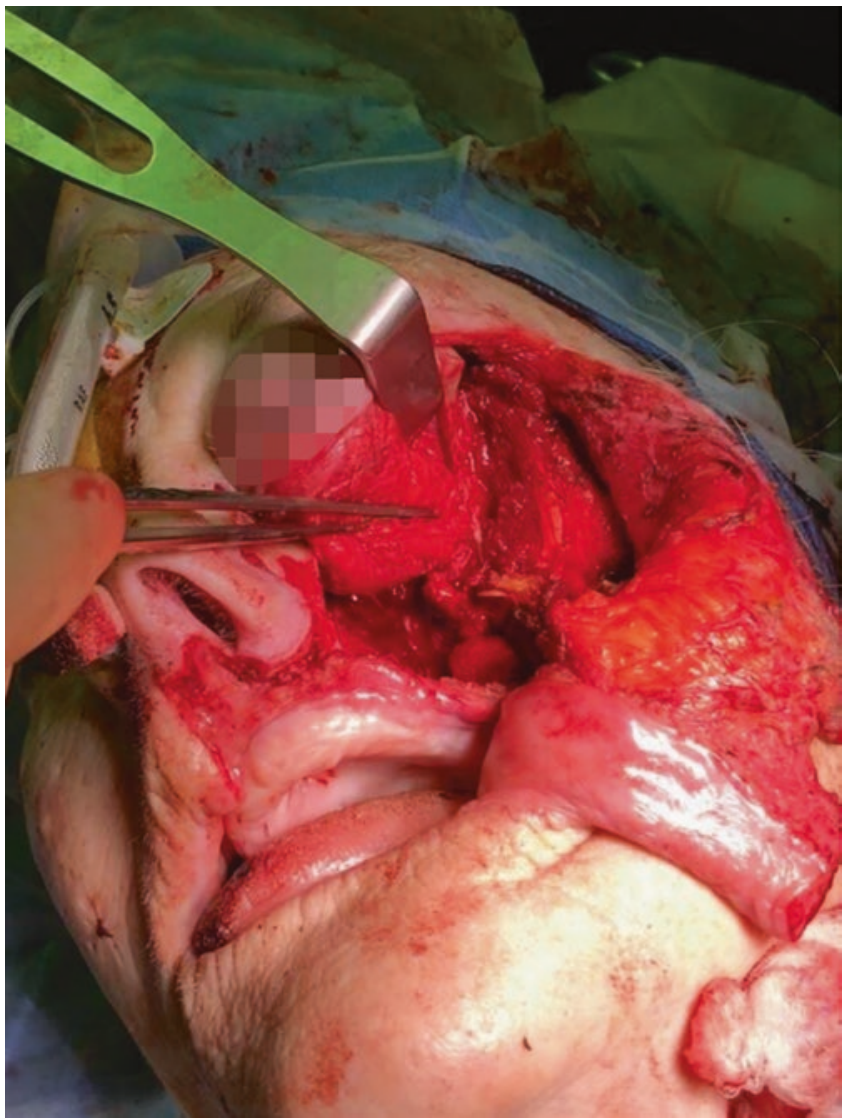

(C)Association of Oral and Maxillofacial Surgeons of India

Fig. 88.3 Osteocutaneous radial forearm free flap for maxillary reconstruction including the orbital rim

\subsubsection{Indications}

Periorbital, nasal and maxillary subunit reconstructions are the common indications for an osteocutaneous radial forearm free flap. It is a real alternative if other donor sites are not viable. 
Fig. 88.4 (a and b) Radial osteocutaneous flap as an alternative in mandible (a, 3D rendering) and (b) maxillary reconstruction (left maxilla CT )
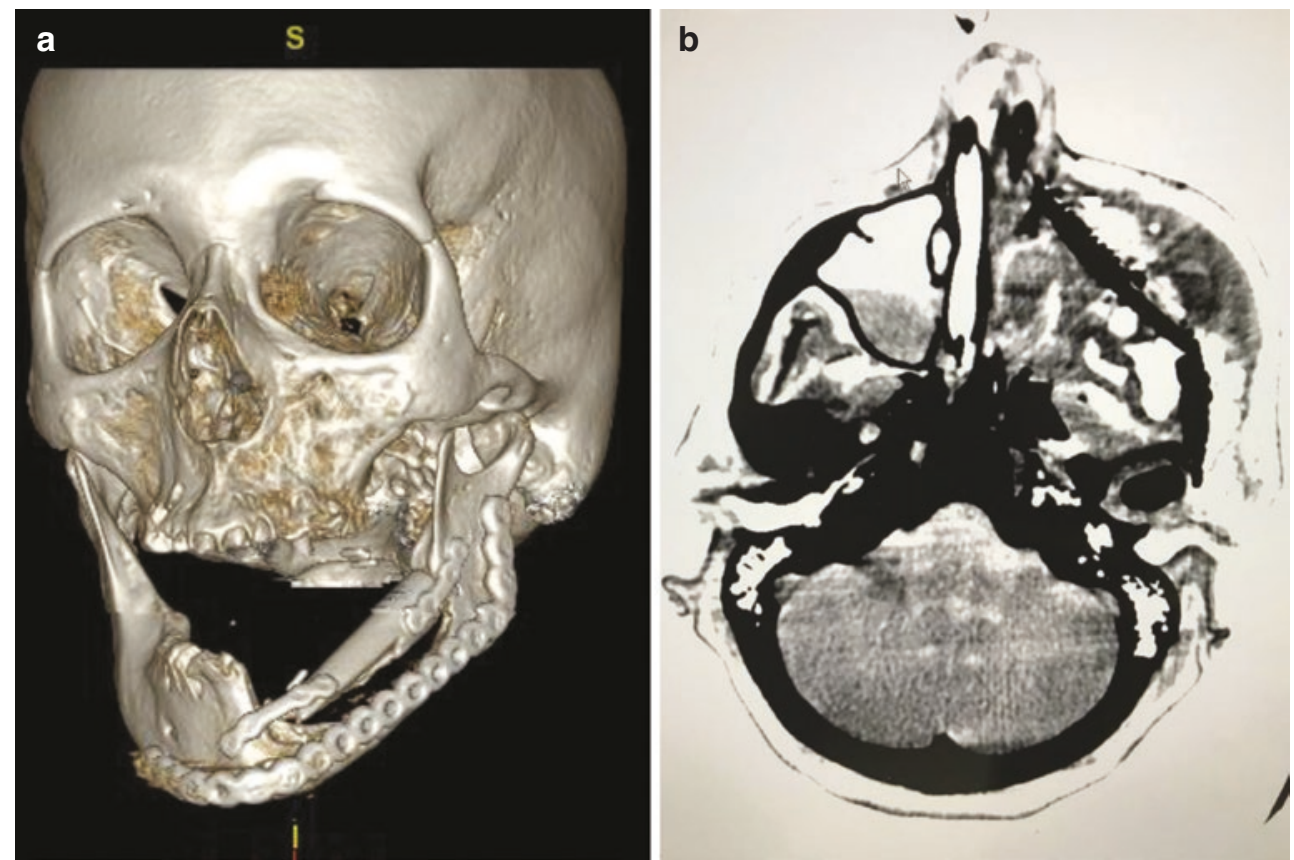

(CAssociation of Oral and Maxillofacial Surgeons of India

\subsubsection{Technique}

Surface markings of the soft tissue from the distal wrist skin crease as the horizontal line, the radial pedicle and the cephalic vein are outlined. The osseous part of the anterior lateral radius segment between the insertion of the pronator teres and the brachioradialis is harvested up to $10-13 \mathrm{~cm}$ in length and about 40 percent in circumference. The distal styloid aspect of the radius should have at least $2 \mathrm{~cm}$ to complete a bone plate fixation after the flap harvest. The fascial and muscular periosteal vascularity is retained in harvest. The flap can be raised from the radial or the ulnar side in a subfascial plane. The brachioradialis is retracted laterally protecting the superficial branch of the radial nerve. A sharp incision is made down to the periosteum preserving the attachment of the intermuscular septum. On the medial side, the flexor carpi radialis muscle is reached and retracted medially so the median nerve is protected. The muscle bellies of the pronator quadratus and the flexor pollicis longus are carefully dissected distal to proximal preserving enough muscular cuff to retain perforators to this segment perfusing from the radial artery in its lateral intermuscular septum. Once the radius periosteum is incised in length noting the curve of radius bone, multiple drill holes are used to outline the osteotomy. A boat-shaped harvest is completed of the cortex protecting the radial vasculature after distal ligation and the muscular perforators to the osseous part. The proximal harvest is completed of the pedicle similar to radial forearm harvest by radial artery and vena comitans ligation. A prebent compression plate is adapted and fixated using bicortical screws across the site of harvest (Fig. 88.5). Osteotomy scan be performed of this

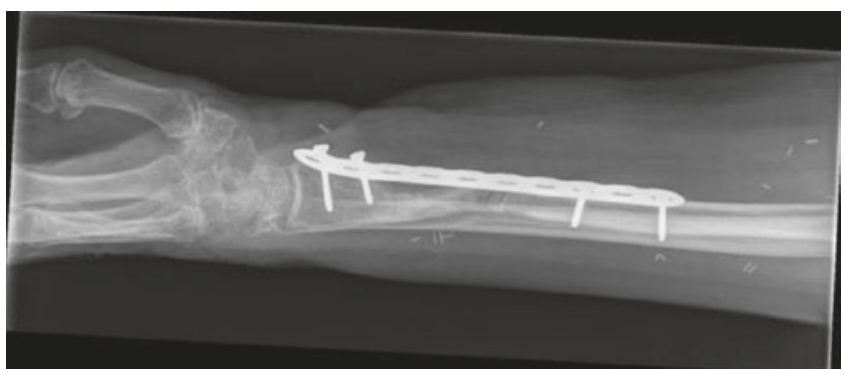

CAssociation of Oral and Maxillofacial Surgeons of India

Fig. 88.5 Post-radial osteocutaneous flap harvest with orthopaedic internal bicortical fixation of radius as prophylaxis for prevention of radial shaft fracture

donor segment with close attention for orbital rim reconstruction if needed.

\subsubsection{Scapula Free Flap [6]}

Theoretical conceptualization of the scapular flap was first described by Saijo in 1978 and popularized by Dos Santos [7] in 1979. This scapular system which got described includes a myriad of options based on the subscapular vessels. It can be used as two types of bony composite flaps with the utilization of angle of the scapula and the lateral border. The major branches of the subscapular system are the circumflex scapular artery and the thoracodorsal artery. Circumflex scapular artery and its associated vein with the lateral border of the scapula are the classical osseous flap. Scapular and parascapular flaps offer the most flexibility 
with combinations of osseous, muscular, fasciocutaneous free tissue transfer based on the subscapular vascular system. Approximately 10-14 cm of the length with a thickness of $0.5-1.5 \mathrm{~cm}$ bone composing the lateral aspect of the scapula and or the angle of the scapula can be harvested.

Figure 88.6 shows the advantage of this flap: independence of soft tissue pedicle in relation to the bone.

It can also be harvested using the angular artery, a branch of the thoracodorsal artery obtaining a longer vascular pedicle and teres major muscle along with the scapular tip. The medial border of the osseous scapular flap has been described; the flexibility of the overlying fasciocutaneous part is not very pliable. The medial border is comparatively narrow even in males for placement of osseointegrated implants.

Tips for donor site confirmation to the defect:

- Contralateral lateral border of scapula for mandibular reconstruction.

- Ipsilateral scapular tip for the palate and maxilla.

- The angle of the scapula can also be used for the mental prominence reconstruction.

Studies have shown good conformance between the scapular tip and the palate. The tip can be used horizontally for a palate and vertically for anterior maxillary reconstruction. There are reports of nonvascular scapular tip being used for orbital floor reconstructions.

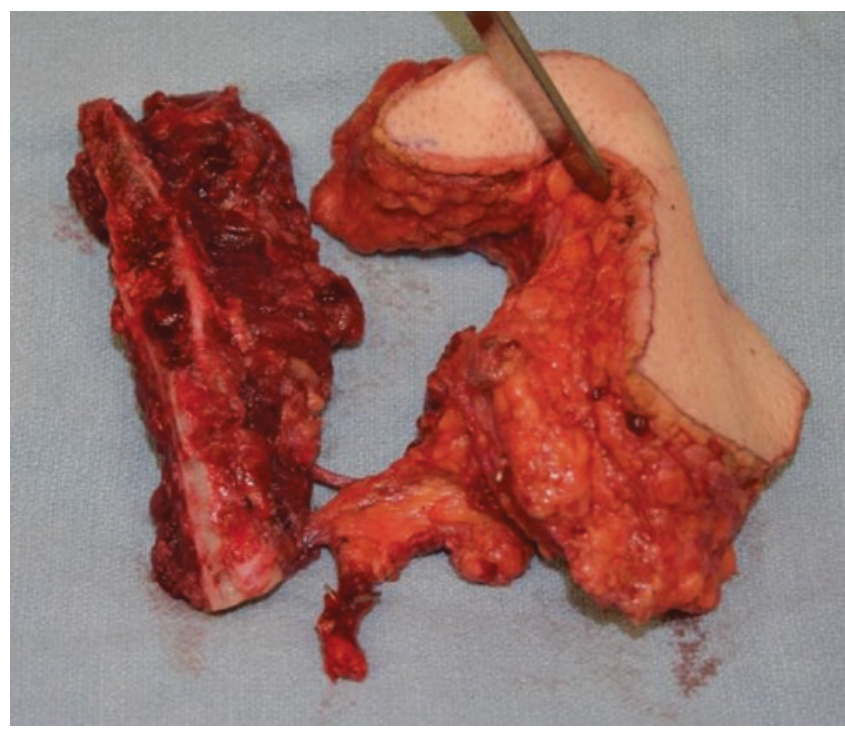

CAssociation of Oral and Maxillofacial Surgeons of India

Fig. 88.6 The osteocutaneous scapular free flap. The great advantage of this flap is the independence of the soft tissue pedicle in relation to the bone

\subsubsection{Indications}

Composite and complex midfacial defects of the orbit, maxilla, skull base and mandible can be reconstructed using this chimeric flap.

\subsubsection{Advantages}

- Minimal donor site morbidity.

- Useful in elderly patients with peripheral vascular disease.

\subsubsection{Disadvantages}

- Concurrent flap harvest during ablation is not possible, as patient needs to be in the lateral decubitus or prone position.

- Inadequacy of the bone stock for osseointegrated implants.

- Variation in the anatomy with the circumflex scapular arising from the axillary artery.

\subsubsection{Technique}

Clear understanding of the scapular arteriovenous system and its branches composing the circumscapular, descending branch and parascapular vessel anatomy is essential.

The osseous branch of the circumflex scapular artery to the lateral border runs directly into it along with the vein. There is a gap between this and the cutaneous perforator which provides the flexibility of the skin paddle.

Patient can be positioned in a lateral decubitus or prone position for access between the axilla to the midline spine. The author's choice is use of a beanbag under the ipsilateral side and a protected axilla for the contralateral side. To increase the flexibility of the pedicle in relation to the lateral border of the scapula, a scapular or parascapular skin paddle can be based on the circumflex artery. The triangular space is made by the teres minor superiorly and teres major inferiorly, and the long head of the triceps laterally is identified using a Doppler $2 \mathrm{~cm}$ superior to the posterior axillary fold edge. The fasciocutaneous flap elevation is medial to lateral superior to the muscular fascia until the omotricipital triangle is reached. The circumflex vascular pedicle can be followed proximally to the subscapular and axillary arterial system with dissection between the teres minor and major muscles superiorly. The circumflex scapular artery is dissected in the lateral aspect of the scapular border with preservation of the muscular perforators to the bone by medial retraction of the teres minor and inferior retraction of the latissimus dorsi. An incision parallel to the lateral border of the scapula is made through the teres minor and infraspinatus muscle and periosteum. Protecting the vascular pedicle laterally on the glenohumeral joint superiorly osteotomy can be performed using a saw to the desired length with or without the angle of the scapula. Scapular angle can be harvested independently with identification of the angular perforators. Adequate care is taken to protect the underlying subscapu- 
laris musculature. Teres major muscle is reattached with multiple drill holes to the new border of the lateral scapula donor site to prevent winging. Perioperative drain and postoperative immobilization of the arm after primary closure of the defect site are commonly utilized along with physiotherapy.

\subsubsection{Fibula Free Flap $[8,9]$}

Taylor et al. first introduced the fibula flap in 1975 for extremity reconstruction but was reintroduced for mandible reconstruction by Hidalgo. Though designed as an osteocutaneous free flap, the initial reports of unreliability of the skin paddle did not find favour in its use as an osteocutaneous flap.

\section{Clinical Pearls}

- Ease of simultaneous two team harvest.

- Consistent anatomy.

- Reliable usable bone length of around $24 \mathrm{cms}$, with the capability of performing multiple osteotomies.

- Variable design as bone only, osteocutaneous or with cuff of flexor hallucis longus or soleus muscle and with accompanying fat and fascia.

- Adequate length of vascular pedicle and suitability of the peroneal vessels for anastomosis.

- Accepts dental implants and osseointegrates.

- Minimal donor site morbidity.

\subsubsection{Anatomy}

The fibula is a triangular bone in cross section, around $40 \mathrm{~cm}$ in length and $1.5-2.0 \mathrm{cms}$ in diameter, articulates proximally with the tibia and distally with the tibia and talus and has dense cortical bone with a small amount of cancellous bone.

Blood supply: Dual endosteal and periosteal. The dominant endosteal nutrient pedicle enters posterior to the interosseous membrane at the junction of the upper and middle thirds of the fibula. It is the segmental periosteal blood supply arising from the peroneal artery that permits multiple osteotomies. Average pedicle length depending on the bone length harvested is from 5 to $10 \mathrm{cms}$ with a diameter of 1.8-2.5 $\mathrm{mm}$ and venous drainage via paired venae comitantes.

Skin paddle: The skin territory for the flap harvest on the lateral aspect of the leg can be up to $25 \mathrm{~cm}$ in length and around $15 \mathrm{cms}$ in width and is supplied by perforators from the peroneal system which can be either septocutaneous or musculocutaneous passing through the flexor hallucis longus and soleus. The number of perforators is around 4-8.

\subsubsection{Preoperative Assessment}

- History of trauma or surgery to the lower limb, any peripheral vascular disease which might coexist.

- Examination of the circulation with the dorsalis pedis and posterior tibial pulse.

- Imaging: MR angiography, CT angiography to rule out peronea magna and also identify perforators. If angiographic studies are contraindicated, perform an arterial and venous duplex ultrasound demonstrating the flow pattern.

Figure 88.7 shows the fibula free flap reconstruction for the left mandibule of the body, angle and ramus. Figure 88.8ac shows the planning and execution for fibula free flap on a stereo lithographic model. The closing osteotomies are made with measurements using a ruler template; the smallest fibula segment is noted as marginally more than 2 centimetres. In Fig. $88.8 b$, one can see a sterile ruler held at the lateral fibula harvest with pedicle attached proximally and skin paddle. In Fig. 88.8c, the osteotomized fibula is immobilized to a reconstruction plate at the lower extremity donor site with the pedicle flowing intact.

\subsubsection{Technique}

Prepare the whole lower limb circumferentially from the calf downwards and the opposite limb for a skin graft. Perform the surface markings of the fibula, with the design of the skin paddle centred along the posterior border that corresponds to the intermuscular septum. Design the skin paddle after the perforators have been mapped out using a Doppler probe. Preserve at least $4-5 \mathrm{~cm}$ bone proximally and distally for the stability of the knee and ankle joint and to avoid injury to the

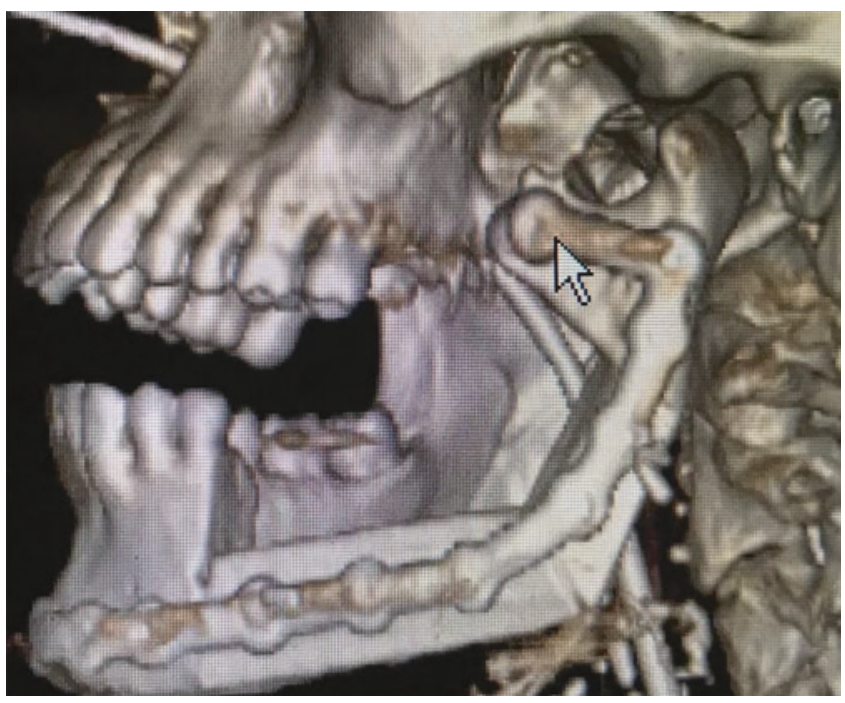

CAssociation of Oral and Maxillofacial Surgeons of India

Fig. 88.7 Fibula free flap reconstruction for the left mandible of the body, angle and ramus 

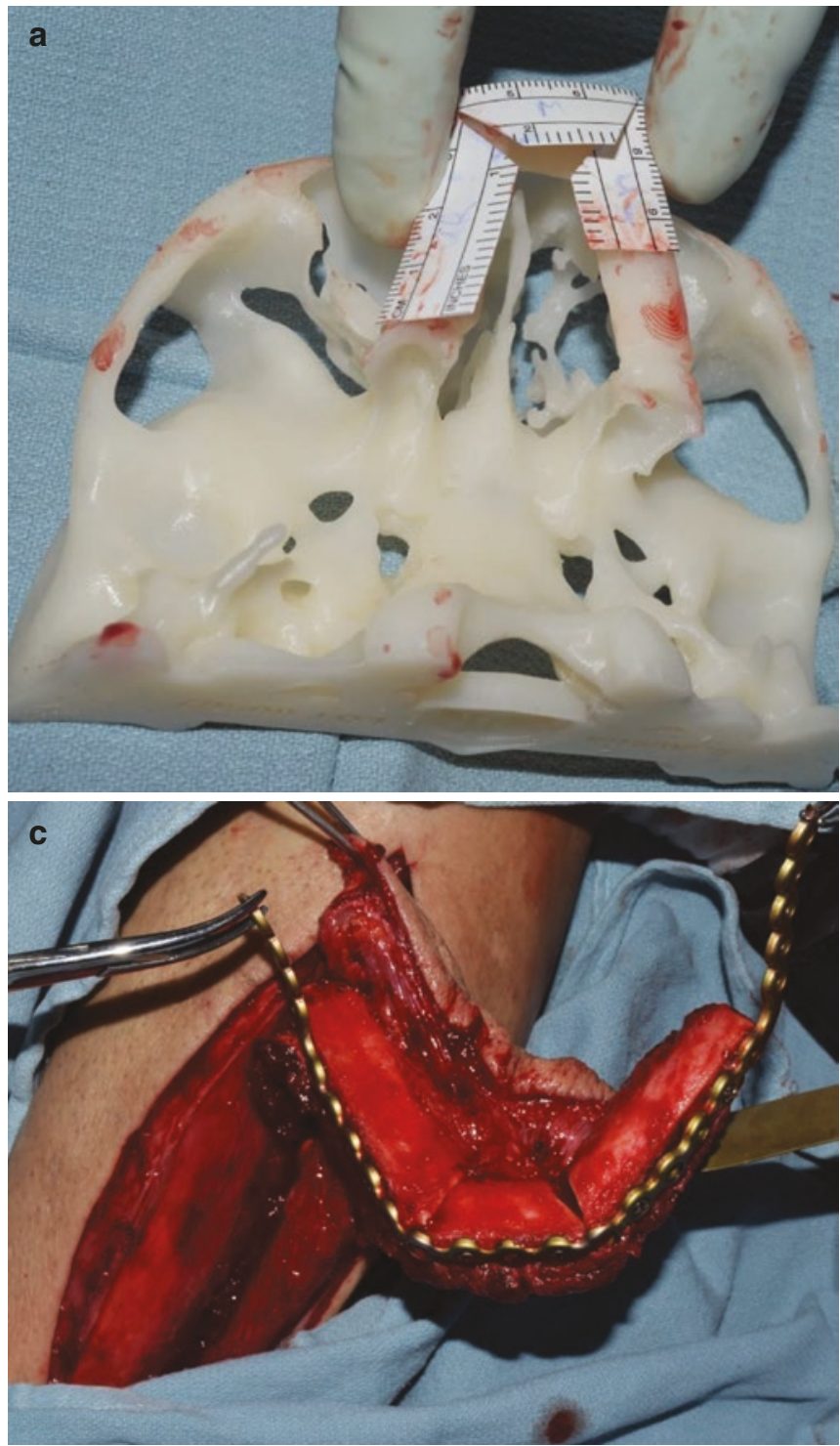

(CAssociation of Oral and Maxillofacial Surgeons of India

Fig. 88.8 Maxillary reconstruction planning for fibula osteotomies. (a) Planning for fibula free flap on a stereo lithographic model. The closing osteotomies are made with measurements using a ruler template; the smallest fibula segment is noted as marginally more than two centimetre. (b), the template being transferred to the fibula area for

superficial peroneal nerve. Carefully apply a tourniquet and exsanguinate the lower limb.

The incision begins anteriorly on the skin paddle deepened into the subfascial plane over the peroneus longus and brevis and dissected to identify suitable perforators. The incision is extended in a curvilinear manner proximally, and dissection in the lateral compartment is done keeping a cuff of peroneus muscle cobblestoned on the bone.

The next step is the dissection in the anterior compartment of the extensor hallucis longus and extensor digitorum

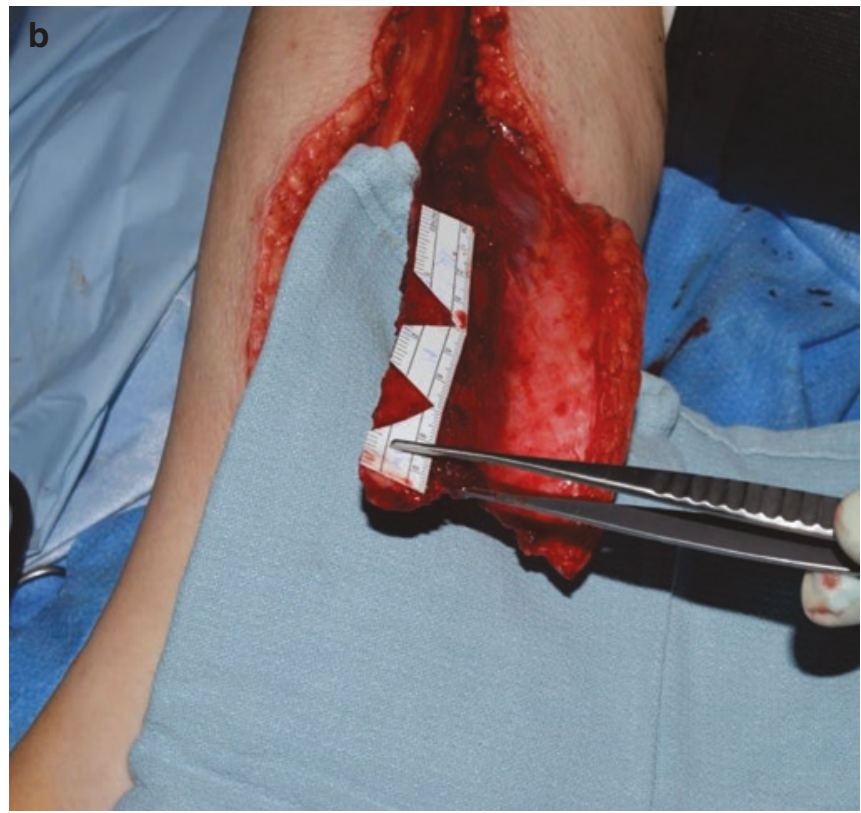

planning the closing osteotomies. You see a sterile ruler held at the lateral fibula harvest with pedicle attached proximally and skin paddle; (c) The osteotomized fibula immobilized to a reconstruction plate at the lower extremity donor site with the pedicle flowing intact

longus up to the dense interosseous membrane which is incised. The anterior tibial vessels are seen anterolateral to the fibula and preserved.

Proximal and distal osteotomies are performed using a reciprocating saw, preserving the superficial peroneal nerve superiorly. Distally ligate the peroneal vessel once the bone is osteotomized.

The posterior compartment dissection is performed between the tibialis posterior and flexor hallucis longus which identify the peroneal vessels. Keep a cuff of flexor hallucis or soleus 
muscle onto the graft depending on the soft tissue requirement and if the skin paddle is centred along the mid bone.

The posterior design of the skin paddle is made in a similar manner deep to the fascia over the soleus and lateral gastrocnemius preserving the saphenous vein and sural nerve.

Identify and preserve the posterior tibial vessels and tibial nerve $1-1.5 \mathrm{~cm}$ medial and parallel to the peroneal vessels.

Perforators to the soleus must be ligated, and additional length of the pedicle can be obtained by a subperiosteal dissection or ligation of the lateral posterior tibial vein.

Shaping of the fibula can be done on the benchside or while still connected with the circulation using either a prebent plate, cutting guides with virtual surgical planning. Closing wedge osteotomies are performed and fixation done.

Donor site closure should be meticulous with good haemostasis; suction drains closing the proximal and distal skin incisions and grafting the remaining donor site. Immobilization with a posterior splint with the ankle in 90 degrees flexion is recommended though not routinely practiced.

Postoperative physiotherapy for the lower limb can commence by the second day and with accompanying gradual weight bearing.

\section{Pitfalls}

- Vascular anomalies and variations can preclude the use.

- A double barrel segment might be needed due to inadequate bone height.

- The soft tissue component may be inadequate for extensive resections.

\subsubsection{Deep Circumflex Iliac Artery-Based Composite Flap or Vascularized Iliac Crest Flap (DCIA)}

Similar to the fibula the DCIA can be planned virtually. Opening osteotomies are performed instead of closing osteotomies in maxilla-mandibular reconstruction (Fig. 88.9a, b, c, d).

This flap can be harvested as an osseous or an osseocutaneous with the soft tissue component of the muscle and skin. This flap is based on the deep circumflex iliac artery and vein with the pedicle length as long as $8 \mathrm{~cm}$ with a diameter of the vessels between 1.5 and $3 \mathrm{~mm}$. The iliac crest osteocutaneous free flap (ICFF) was described separately by Taylor [10] et al. and Sanders and Mayou in 1979 and popularized by Urken in 1989 for mandibular reconstruction and by Brown [11] in 1996 for maxillary reconstruction and use of internal oblique muscle [11]. The deep circumflex iliac artery and vein vessels arise from the external iliac vessels. The bone quality and quantity are excellent for both the maxillary as well as mandibular reconstruction.

Maxilla and mandibular segments can be reconstructed using the iliac crest bone based on the deep circumflex branch of the external iliac artery. The authors prefer a harvest using the superior approach in reference to the spermatic cord or the round ligament. Donor site morbidity is not significant, and the patient can be supine allowing a two-team approach. Up to $14 \mathrm{~cm}$ of bicortical bone with internal oblique muscle can be harvested. The thickness can vary between $0.5 \mathrm{~cm}$ and $2.5 \mathrm{~cm}$.

\subsubsection{Indications and Contraindications}

This composite flap offers optimal volume of bone for endosteal implants as well as segmental mandibular defects with need of reconstruction of the dentate contour. Maxillary palatal contour and the regional complex subunits after maxillectomy can be reconstructed. The relative contraindications for this harvest are inguinal hernia, obesity, hip prosthesis and polyparity in women.

\subsubsection{Vascular Anatomy}

Just above the inguinal ligament, the deep circumflex iliac artery (DCIA) branches off the posterolateral aspect of the external iliac artery. As it traverses laterally parallel to the inguinal ligament, the ascending branch takes off 1-2 cm medial to the anterior superior iliac spine. This is located between the internal oblique on the transversalis fascia with penetrating perforators. The DCIA has a diameter averaged at $2 \mathrm{~mm}$ and a pedicle length of 5-6 cm. Vena comitans accompanies the artery draining into the external iliac vein. The position of the DCIA is about $2 \mathrm{~cm}$ inferior to the surface of the iliac crest at the fusion of the transversalis fascia and iliacus muscle. The two variations reported are the ascending branch takeoff in medial relation to the anterior superior iliac spine $(1-2 \mathrm{~cm}$ versus 2-4 cm) and multiple ascending branch perforators instead of a single identifiable ascending branch of the DCIA.

\subsubsection{Technique}

With the patient supine, a soft hip support is placed and the skin prepped from the midline pubis laterally to the midaxillary line, superiorly from the subcostal margin to the anterior thigh inferiorly with the greater trochanter included in the field. This includes the planned cutaneous paddle. The cutaneous perforators are concentrated $5 \mathrm{~cm}$ lateral to the anterior superior iliac spine and $4 \mathrm{~cm}$ superior to the iliac crest midpoint. Only internal oblique musculature for lining of the oral mucosal lining is included. Incision is made in a superomedial elliptical fashion through the Scarpa's and Camper's fascia up to the external oblique musculature. This is identified in its orientation from the lateral to medial direction, and as this is incised, the underlying 90 degree medial to lateral internal oblique muscle is identified. The external oblique musculature is included with the osteocutaneous 

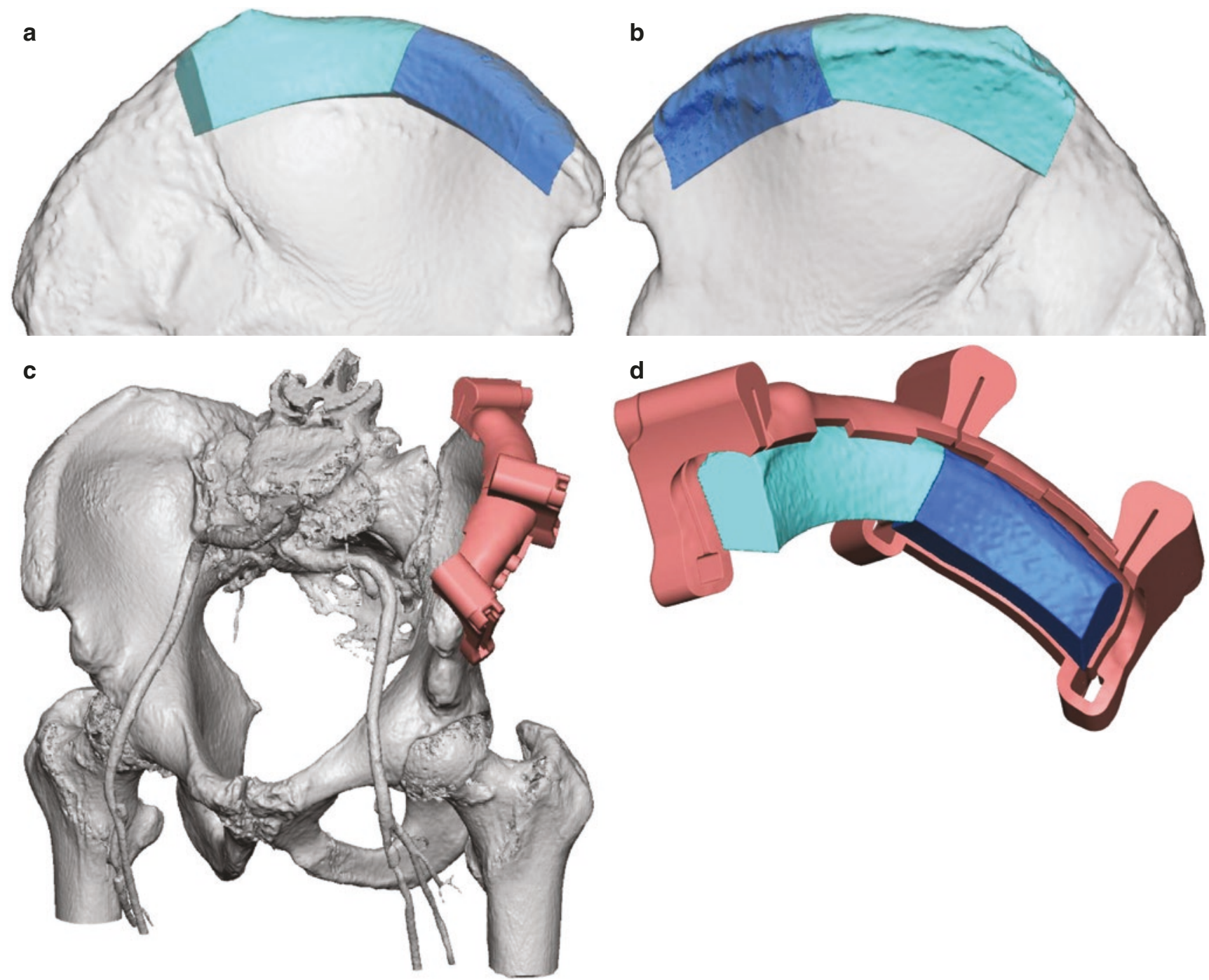

(C)Association of Oral and Maxillofacial Surgeons of India

Fig. 88.9 (a, b, c, d) Similar to fibula DCIA can be planned virtually. Opening osteotomies are performed instead of closing osteotomies in maxilla-mandibular reconstruction

flap. The external oblique incision extends from the lateral entirety of the whole length up to the border of the inguinal ligament. The superior part of the flap is retracted and the internal oblique muscle exposed, and the amount of this internal oblique muscle inclusion is identified for harvest superior to the iliac crest attachment. The internal oblique and transversalis fascia is dissected sharply from the superior lateral aspect to the inferior lateral direction with superior retraction and access. The ascending branch is visualized and dissection is directed to identify the DCIA itself. The ileacus muscle is visualized and preserved based on the pedicle position and harvesting about $2 \mathrm{~cm}$ of it. At least $4 \mathrm{~cm}$ of the internal oblique muscle is harvested lateral to medial towards the ilioinguinal ligament. The vein runs over the DCIA medially to the external iliac vein.

The bone harvest is performed after detaching the gluteus medius muscle from the outer aspect of the ilium. The medial bone cut is made depending on the inclusion of the anterior crest of the ilium. The template as shown in the figure (anterior maxillary virtual finding figure) is used for making medial and lateral cuts using an oscillating saw. The medial side should be protected for pedicle integrity and peritoneum. If osteotomies are needed of the bony pedicle, they're performed with adequate care to gently greenstick the components.

The abdomen is closed in layers after adequate haemostasis of the donor osseous margins of the osteotomies. Multiple postage stamp holes are made in the bicortical iliac cortex and the residual muscular layers reattached with thicker nonresorbable sutures. And non-resolvable mesh can be used to reinforce the harvested internal oblique defect, and this can be sutured to the cortical holes as described above. The cutaneous closure is performed over suction drainage with multiple layer closure. 
The quality and quantity of the bone of this flap are ideal for maxillofacial defect reconstruction. Maxillary reconstruction may need a rather lateralized bony segment harvest to gain adequate pedicle length for anastomosis into the neck. If the mandibular angle is to be reconstructed, the anterior iliac crest is included. An entire hemimandible can be reconstructed using the unilateral iliac crest.

\subsubsection{Vascularized Rib Graft}

There are other osteocutaneous flaps which are described like posterior costal osteocutaneous flap similar to the harvest of the costochondral part of the rib. These vascularized grafts can be from the right fifth rib in the ventral surface and up to the ninth rib in the dorsal surface. The intercostal vascular pedicle and subcutaneous venous pedicle or even the internal mammary perforators medially are used. The ribs can also be a part of the pectoralis major osteomyocutaneous flap, latissimus dorsi, serratus and trapezius muscular harvest with their pedicles. Of course the isolated rib graft with or without the costochondral graft has been the work horse for temporomandibular reconstruction for decades. The harvest has been documented well in surgical literature over the years.

\subsubsection{Lateral Femoral Condyle Free Flap}

The choice of this flap is for novelty in maxillofacial surgical needs. Femoral internal condyle with the descending genicular artery and vein as a pedicle can be harvested as free flap. The bony corticocancellous segment measuring up to $8 \times 1.5 \times 1.5 \mathrm{~cm}$ can be harvested [12].

\subsection{Conclusion}

In conclusion the hard tissue regeneration and reconstruction have been a primary focus in clinical and research interests for all reconstructive maxillofacial surgeons. The techniques and molecular science have ongoing updates persistently for the optimal and ideal technique. The understanding of intercellular and molecular behaviour of regeneration and grafting procedure is sought by biomedical scientists by research. And with virtual surgery and planning with guided navigation, the future of hard tissue reconstruction is poised for technically predictable results.

\section{References}

1. Boyne PJ, Sands NR. Secondary bone grafting of residual alveolar and palatal clefts. J Oral Surg. 1972 Feb;30(2):87-92.

2. Marks R, Stevens M. Atlas of oral and extraoral bone harvesting. Hanover Park, IL: Quitessence; 2010.

3. Abubaker O, Oghalai M. Selected readings in oral and maxillofacial surgery. $2001 \mathrm{Dec} ;$ 9(6).

4. Melville JC, Shum JW, Chandra SR. Chapter 12: tissue engineering for transoral reconstruction of large mandibular continuity defects. In: Melville JC, Shum JW, Young S, Wong ME, editors. Regenerative strategies for maxillary and mandibular reconstruction: a practical guide. Cham: Springer; 2019. p. 143-54.

5. Catone G, Reimer B, Mcneir D, Ray R. Tibial autogenous cancellous bone as an alternative donor site in maxillofacial surgery: a preliminary report. J Oral Maxillofac Surg. 1992;50:1258-63.

6. Swartz WM, Banis JC, Newton ED, Ramasastry SS, Jones NF, Acland R. The osteocutaneous scapular flap for mandibular and maxillary reconstruction. Plast Reconstr Surg. 1986;77(4):530-45.

7. Dos Santos LF. Un novo retalho livre microcircugio. Rev Bras Cit. 1980;70:133.

8. Taylor GI, Miller GD, Ham FJ. The free vascularized bone graft. A clinical extension of microvascular techniques. Plast Reconstr Surg. 1975;55(5):533-44.

9. Hidalgo DA. Fibula free flap: a new method of mandible reconstruction. Plast Reconstr Surg. 1989;84(1):71-9.

10. Taylor GI, Townsend P, Corlett R. Superiority of the deep circumflex iliac vessels as the supply for free groin flaps. Plast Reconstr Surg. 1979;64(5):595-604.

11. Brown JS. Deep circumflex iliac artery free flap with internal oblique muscle as a new method of immediate reconstruction of maxillectomy defect. Head Neck. 1996;18(5):412-21.

12. Martin D, Bitonti-Grillo C, De Biscop J, Schott H, Mondie JM, Baudet J, Peri G. Mandibular reconstruction using a free vascularised osteocutaneous flap from the internal condyle of the femur British. J Plast Surg. 1991;44:397-402.

\section{Suggested Reading List}

1. Langdon JD. Operative Oral and Maxillofacial Surgery. second ed. London: Hodder Arnold: 2009.

2. Coleman III JJ. Plastic Surgery Indications, Operations and Outcomes. Volume Three Head and Neck Surgery. first ed. St Louis: Mosby: 2000.

3. Wolff KD. Raising of Microvascular Flaps. A Systematic Approach. second ed. Berlin: Springer: 2011.

4. Cheney ML. Facial Surgery Plastic and Reconstructive. second ed. FL: CRC Press Taylor \& Francis Group: 2015.

Open Access This chapter is licensed under the terms of the Creative Commons Attribution 4.0 International License (http://creativecommons. org/licenses/by/4.0/), which permits use, sharing, adaptation, distribution and reproduction in any medium or format, as long as you give appropriate credit to the original author(s) and the source, provide a link to the Creative Commons license and indicate if changes were made.

The images or other third party material in this chapter are included in the chapter's Creative Commons license, unless indicated otherwise in a credit line to the material. If material is not included in the chapter's Creative Commons license and your intended use is not permitted by statutory regulation or exceeds the permitted use, you will need to obtain permission directly from the copyright holder. 